\title{
Proposal of Mechatronic Devices Control using Mixed Reality
}

\author{
Erich Stark, Erik Kučera, Peter Drahoš, Oto Haffner \\ Slovak University of Technology in Bratislava \\ Bratislava, Slovakia \\ Email: erich.stark@stuba.sk
}

\begin{abstract}
The Internet of Things and mixed reality are now among the most important areas in research or in practice. The aim of this paper is to propose an appropriate way of connection of these two areas, where is possible to control and monitor mechatronic devices using a mobile device with augmented/mixed reality support. The main task will be to explore these options in the area and implement this solution as prototype. The proposed methodology for control and diagnostics of mechatronic devices is modern as it combines hardware management, a Unity3D engine for mixed reality development, and communication within the Internet of Things network.
\end{abstract}

\section{INTRODUCTION}

C URRENTLY, computer networks are no longer just for connecting conventional computers like they once were. Their purpose gained a new dimension when mobile devices and embedded systems began to connect to these networks. At present, these boundaries are shifted to the level of connection of individual sensors, various household appliances, and even autonomous cars to the network [1]. This expansion of connected devices has also happened because of the rise of microcomputers like Raspberry Pi, DragonBoard, and similar prototyping solutions. At the same time, people begin to realize the value of data that these sensors generate. They can help us streamline processes in industry and services or make life easier with smart home solutions. As a result, the emergence of new types of networks such as Internet of Things (IoT) are needed. The concept of IoT can be found at almost every conference in the field of information and communication technologies or in scientific articles [10], [11], [12]. The Gartner company makes regular analyzes and research into the use of various technologies. Earlier in 2017 , an analysis was made that states that IoT will have up to 20.5 billion connected devices in 2020 [2]. These paradigms would not take place without the development of new networks, data transmission protocols and the necessary software tools. At present, IoT devices are controlled by console, web, or mobile applications. Using these conventional methods of controlling IoT devices in a small room can be quite simple. Because the list of devices is on one screen, we can see and set properties almost instantly. But if there are multiple rooms or buildings, the segmentation of these devices may be totally unclear and cumbersome. Here is the opportunity to use current trends and modern technologies in the field of virtual, augmented and mixed reality. These technologies are able to put digital objects into the real world. Their convenience lies in the fact that objects from the real world are enriched with information relevant to the given object that one is looking at. This camera stream processing is real-time. Mixed reality can now be developed and tracked with compatible headset - such as Microsoft HoloLens or compatible mobile devices (smartphones and tablets) from both leaders in the segment - Google Android and Apple iOS. The implementation of the proposed project involves the use of mobile devices for their wide availability - whether for household or industry. Compatible headsets are currently not suitable for this purpose, as businesses (especially small and medium-sized ones) are often unwilling to invest in these headsets. The proposed methodology for controlling and diagnosing IoT devices is modern as it combines hardware management, a 3D engine for mixed reality development, and communication within the Internet of Things network - all areas of mechatronics. The proposed solution is unique and will contribute to the scientific field of mechatronics.

\section{Computer Generated Reality}

\section{A. Virtual Reality}

Virtual reality (VR) is a term that is mentioned in various areas, not only in information and communication technologies. Films like Matrix have brought virtual reality from the sci-fi world to the human mind. Examples of virtual and extended reality are becoming more and more real-life, from military air simulators to simple smartphone applications. Everyone can have their own idea of virtual reality, so it is necessary to introduce a suitable definition.

Virtual reality consists of an interactive computer simulation that senses the state of the user and replaces or extends sensory feedback information to one or more senses in such a way that the user gets a feeling of being immersed in the simulation (virtual environment).

\section{B. Augmented Reality}

Augmented reality (AR) is an overlapping of content in the real world, but this content is not embedded or part of it. The content of the real world is not capable of responding to computer-generated content [3]. Augmented reality is therefore a live, direct or indirect view of a real world that is complemented by computer generated (CG) elements such as audio, video, graphics, or GPS data. Augmented reality is a layer of content above the real world, and this content is not 
anchored to this world or its part. As has been said, elements of the real world and CG content can not react with each other.

The purpose of Augmented reality is to improve user perception and improve its effectiveness through additional information. The user retains awareness of the real world, but in an ideal extended reality it would not be able to recognize the difference between information from the real world and the virtual world.

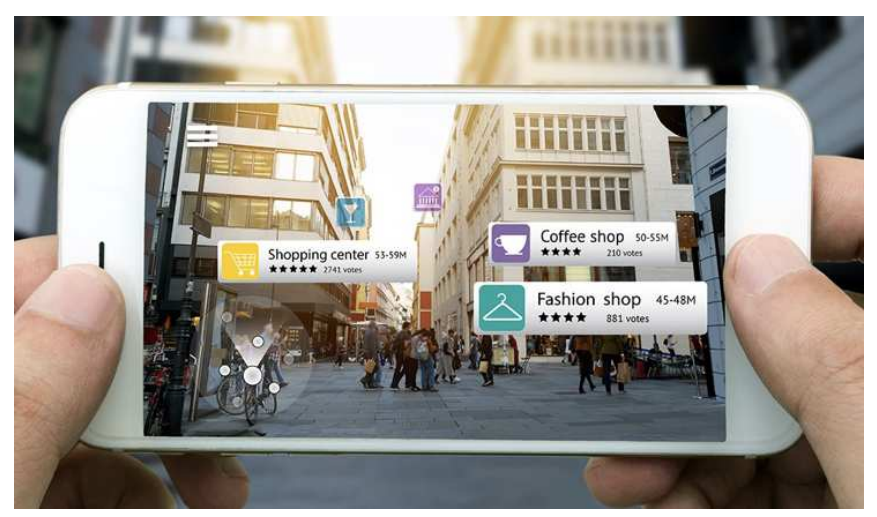

Fig. 1. Augmented reality example

\section{Mixed reality}

Mixed reality (MR) is an overlap of the real world with synthetic content that is embedded in it and interacts with the real world. The key feature of MR is that real-world synthetic content and content can respond in real time to one another. Mixed reality is thus a combination of the real world and the virtual world, creating a new environment and visualization where physical and digital objects coexist and interact with each other in real time. Mixed reality is the layer of artificial (digital) content in a real world that is anchored and interacts with the real world. An important fact is that, in the case of mixed reality, advanced mapping of the environment is required for the placement of additional $\mathrm{CG}$ elements.

If information is to be successfully combined, virtual objects must act physically in a suitable way. If a real and virtual object collision occurs, both must respond appropriately. In addition, virtual objects must overlay the view of real objects and also shadow on them. All this can only be achieved by a precise model of real and virtual environments.

The first hardware for mixed reality, currently the most advanced device of the segment, is Microsoft HoloLens. The problem is still a relatively high price, but there is also an emulator for development.

Based on the information we have mentioned, mixed reality seems to be the most exciting. However, it is possible to imagine the future in which synthetic content will be able to react in some way and even communicate with the real world [3].

\section{INTERNET OF THINGS}

Internet of Things is currently a very widespread term in the field of modern information and communication technologies.

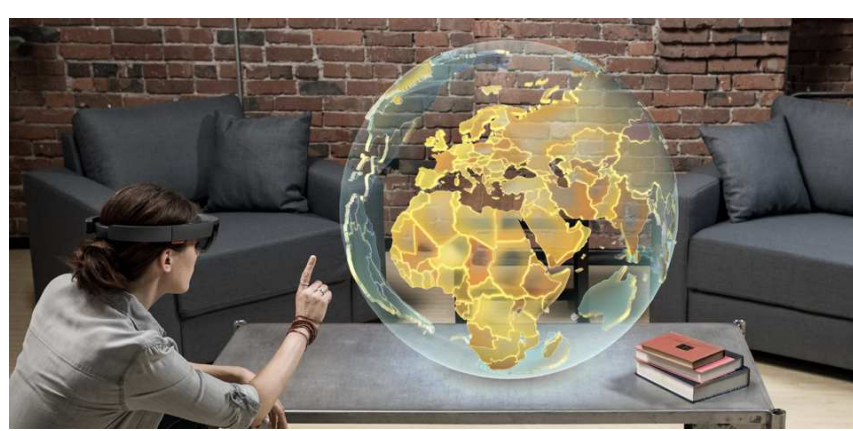

Fig. 2. Mixed reality example

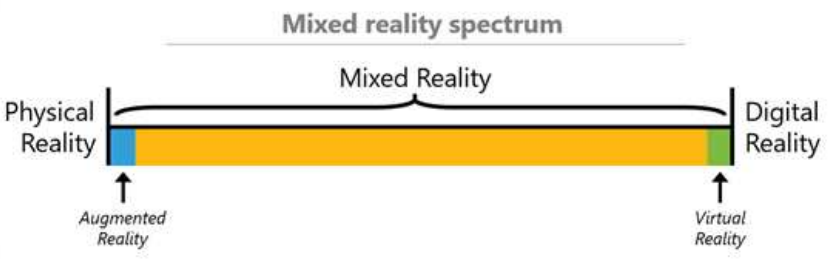

Fig. 3. Mixed reality spectrum

This issue is the subject of various debates as its deployment in industry and services brings about more effective action, but it also raises various issues, such as safety. Thus, IoT concerns almost all fields of human activity [4].

In general, IoT can be defined as a set of physical objects (or things) embedded in electronics, software, sensors and connected devices that are connected together in the network to allow data exchange with other interconnected devices to achieve higher value and more services for users. These IoT devices create a linked network in which each is uniquely identifiable with a unique IP address and capable of communicating with existing network infrastructures.

\section{RELATED WORK}

In the paper [5], the authors presented the Augmented Things concept, where computer objects contain all the information needed to track and expand the information required by AR applications. This allows the user to connect to them, retrieve information using their mobile device, and get expanding information like, for example, maintenance, device, or usage information. The authors have created also a simple 3D framework that allows you to track objects using high quality 3D high resolution scans.

Phillipe Lewicki has attempted to create a demonstration program to help control the Philips Hue light bulb using the Microsoft HoloLens device as seen in Fig. 5. He realized that today's solutions allow you to control the bulbs using the mobile application they need to open, to find a particular room, and then a particular bulb. Often, such applications are limited because smart bulbs contain more features than just turn on / off.

Thanks to the HoloLens on the head, it was only possible to look at the light and turn it into a simple gesture or change 


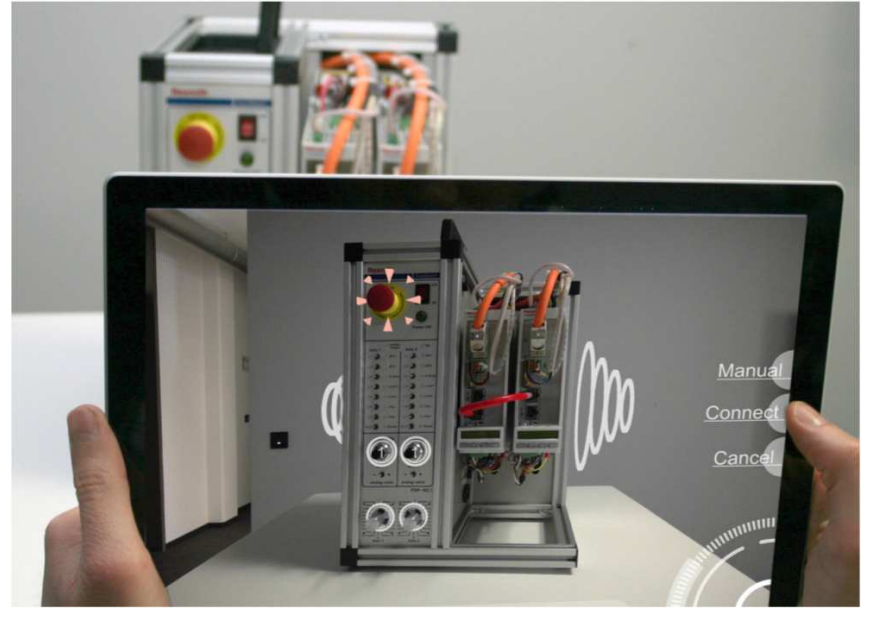

Fig. 4. Augmented Things objects contain and share their AR information

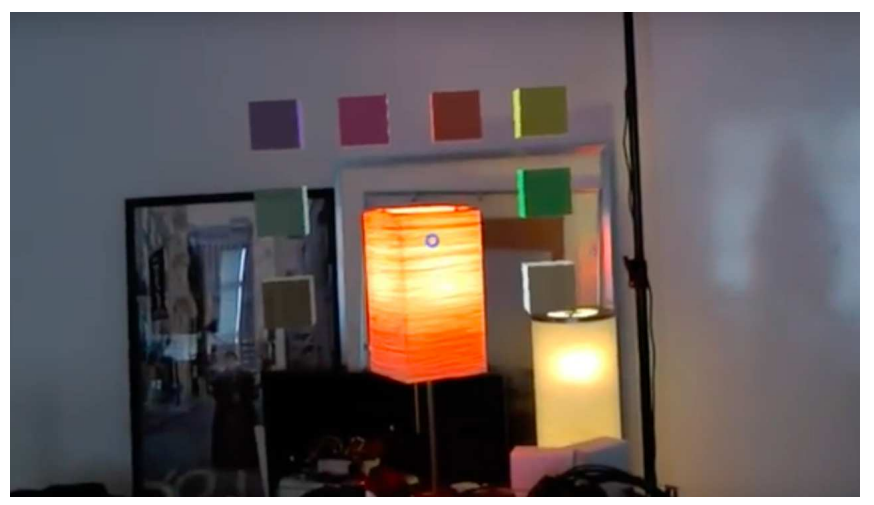

Fig. 5. Application for HoloLens, enabling color adjustment of light

the color of the light. It was faster than a wall switch [6]. Fig. 6 shows Proof of Concept (PoC) by designer Ian Sterling and software engineer Swaroop Pala. Their concept shows how smart devices could be controlled by gestures. The task of this project was to provide a 3D user interface with Android Music Player and Arduino light fan [7].

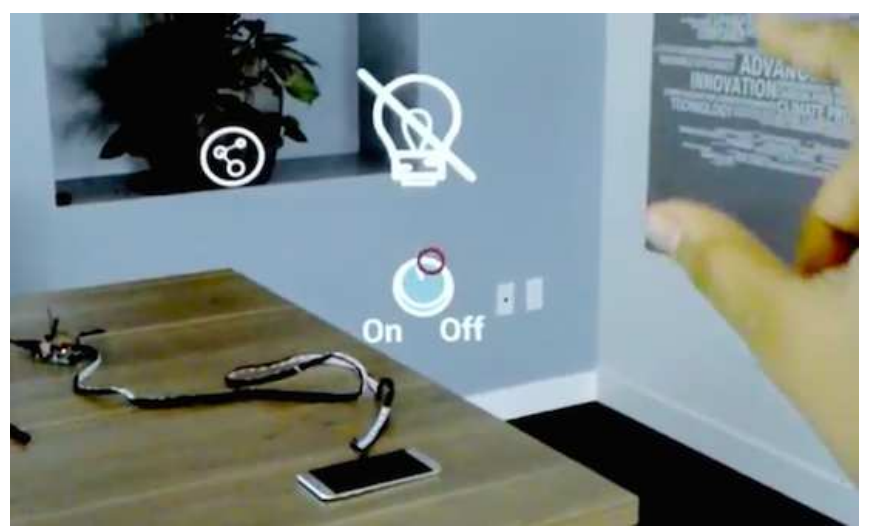

Fig. 6. Light control using HoloLens and Arduino devices

\section{SYSTEM PROPOSAL}

The diagram of the proposed system can be seen in the Fig. 7.

System description:

1) At the beginning of the system is mixed reality device which is able to analyze data stream from camera and detects QR code.

2) Application can connect to identical object in the cloud.

3) Data from the device sensors are sent to the cloud.

4) Mixed reality application gets information about device and shows tailored user interface.

5) The user can interact with that device using mixed reality experience.

6) It is possible to send some control commands to the cloud.

This system can be decoupled into several components described in subsections below.

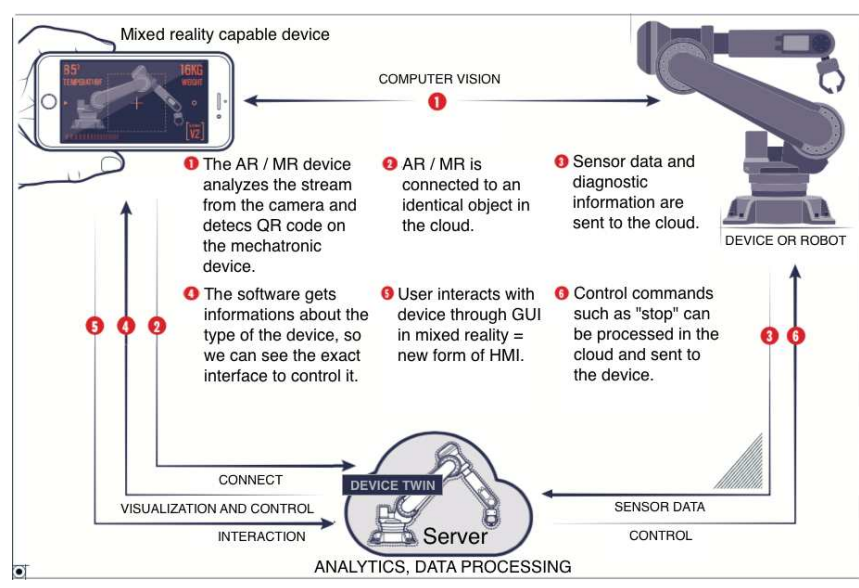

Fig. 7. Diagram of system proposal

\section{A. Camera Device with Mixed Reality Support and Computer Vision Tools}

The best option for mixed reality experiences nowadays is smartphone when comes to price or availability for masses. Basically, there are two options: Android system with ARCore SDK or iOS with ARKit SDK support. ARKit SDK was chosen because of greater support of functions needed for this project. For example, like persistent content or 3D object recognition in recently announced ARKit 2.

At first it has to be created software which can analyze video stream from smartphone camera and detects physical objects. ARKit can be used with support of framework Vuforia inside Unity 3D editor, which helps a lot with software development. The main features of Vuforia SDK is Multi Target detection, User Defined Targets or Cloud Recognition.

\section{B. Software Platform for IoT}

OPC is currently the most advanced standardized data exchange process for automation technology. It allows the collection and transmission of data in a unified form from 
various devices, control systems and applications throughout the organization. The design of this standard allows mapping almost all industrial data into the OPC data structure. OPC UA is an enhanced version of the OPC standard that has a unified architecture that makes it a platform-independent protocol. In addition, it has built-in security mechanisms and applications are fully scalable from microcontrollers to corporate servers.

\section{IoT Prototyping Hardware Kit}

There were many options for prototyping devices from single hardware to complex IoT kits. It was not easy to find IoT kit which meet system requirements the most.

BigClown is a modular hardware and software system that allows to prototype and build real-world telemetry, automation and other applications including IoT. BigClown can be imagined as a set of components with a single interface that can be connected together depends on application needs.

The core of each device is the so-called Core Module. It is powered by a single core CPU with the Cortex-M0+, specifically STM32L083CZ. This chip was chosen for a number of reasons: it is proven and used ARM CPU from STM32 series, has a very low consumption (which is important for powering the nodes from battery), has integrated USB with ROM bootloader, enough number of interfaces, (Flash, RAM and EEPROM), and above all, it has two cryptographic components: TRNG (True Random Number Generator) and AES-128 computing accelerator [8].

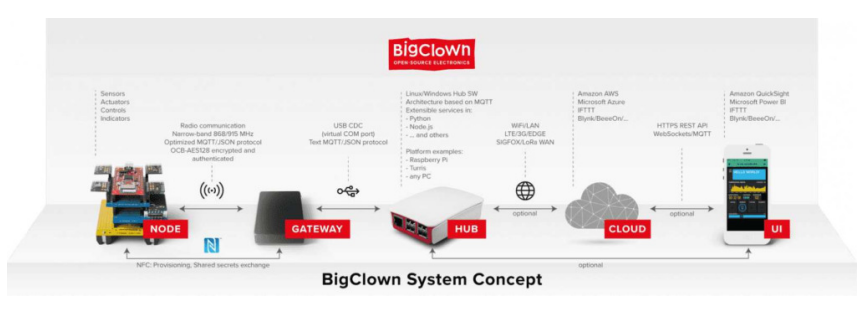

Fig. 8. Whole BigClown ecosystem [8]

\section{CONCLUSION}

The upcoming trend Internet of Things has an impact not only on applications for various services, households and intelligent buildings, but also significant impact on industry and industrial production. The application of IoT principles in industry is called Industrial Internet of Things (IIoT), where in this case instead of interconnected devices is used individual machine parts or their sensors and actuators, as well as sensors and actuators for HVAC (Heating, ventilation, and air conditioning) security. Device interconnection should be wireless in particular and should bring new interaction capabilities not only between systems, but also bring new capabilities to control, track and secure advanced services.
This proposal of interconnection IoT and mixed reality can bring new form of Human Machine Interface which can save time for users or companies.

The future work will focus on developing such a solution with technologies mentioned before.

\section{ACKNOWLEDGMENT}

This work has been supported by the Cultural and Educational Grant Agency of the Ministry of Education, Science, Research and Sport of the Slovak Republic, KEGA 030STU4/2017 and KEGA 038STU-4/2018, and by the Young researchers support program, project No. 1324 - VZRI4 (Virtual and Mixed Reality for Industry 4.0) and project No. 1325 ODIZPZR (Monitoring and Diagnostics of IoT Devices using Mixed Reality).

\section{REFERENCES}

[1] Hammar, Sven. Connected cars: Driving the Internet of Things revolution: How to run an IoT enabled business [online]. IoT Now, 2017 [cit. 2018-03-23]. Available at: https://www.iot-now.com/2017/04/03/60270connected-cars-driving-internet-things-revolution/

[2] Meulen, Rob van der. Gartner Says 8.4 Billion Connected "Things"Will Be in Use in 2017, Up 31 Percent From 2016: IoT Units Installed Base by Category [online]. Gartner, 2017 [cit. 2018-03-23]. Available at: https://www.gartner.com/newsroom/id/3598917

[3] Virtual Reality: $V R, A R, M R$ [online]. The Foundry [cit. 2018-0422]. Available at: https://www.foundry.com/industries/virtual-reality/vrmr-ar-confused

[4] Pohanka, Pavel. Internet veci [online]. i2ot, 2017 [cit. 2018-03-25]. Available at: http://i2ot.eu/internet-of-things/.

[5] Rambach, Jason et al. Augmented Things: Enhancing AR Applications leveraging the Internet of Things and Universal 3D Object Tracking. In: IEEE International Conference on Industrial Technology (ICIT). 2017, zv. 22, s. 25.

[6] Lewicki, Philippe. Controlling lights with the Hololens and Internet of Things [online]. htmlfusion, 2016 [cit. 2018-04-03]. Available at: http://blog.htmlfusion.com/controlling-lights-with-the-hololensand-internet-of-thingsatch-one-of-philippes-appearances-in-june/

[7] Sterling, Ian a PAL, Swaroop. Control with your smart devices by staring and gesturing [online]. Arduino, 2016 [cit. 2018-0403]. Available at: https://blog.arduino.cc/2016/07/26/control-with-yoursmart-devices-by-staring-and-gesturing/

[8] Maly, Martin. BigClown: IoT jako modulární stavebnice [online]. Root.cz [cit. 2018-04-22]. Available at: https://www.root.cz/clanky/bigclown-iot-jako-modularni-stavebnice/

[9] T. Pałys, K. Murawski, A. Arciuch and A. Walczak, "Optical driving for a computer system with augmented reality features," 2017 Federated Conference on Computer Science and Information Systems (FedCSIS), Prague, 2017, pp. 657-662.

[10] M. P. Loria, M. Toja, V. Carchiolo and M. Malgeri, "An efficient realtime architecture for collecting IoT data," 2017 Federated Conference on Computer Science and Information Systems (FedCSIS), Prague, 2017, pp. 1157-1166.

[11] R. Falkenberg et al., "PhyNetLab: An IoT-based warehouse testbed," 2017 Federated Conference on Computer Science and Information Systems (FedCSIS), Prague, 2017, pp. 1051-1055.

[12] J. Mocnej, T. Lojka and I. Zolotová, "Using information entropy in smart sensors for decentralized data acquisition architecture," 2016 IEEE 14th International Symposium on Applied Machine Intelligence and Informatics (SAMI), Herlany, 2016, pp. 47-50. 